\title{
New Particle Formation from the Vapor Phase : From \\ Barrier-Controlled Nucleation to the Collisional Limit
}

\section{Dingilian, Kayane K.}

2021-05-20

pÿDingilian , K K , Lippe , M , Kubeka , J , Krohn , J , Li , C , Halonen , R , Keshavarz , F , Reischl , B , Kurtén , T , Vehkamäki , H , Signorell , R \& Wyslouzil , B E 2021 , ' New Particle Formation from the Vapor Phase : From Barrier-Controlled Nucleation to the Collisional Limit ' , Journal of Physical Chemistry Letters , vol. 12 , no. 19 , pp. 4593-4599 . https://doi.org/10.1021/acs.jpclett.1c0076،

http://hdl.handle.net/10138/330164

https://doi.org/10.1021/acs.jpclett.1c00762

cc_by_nc_nd

publishedVersion

Downloaded from Helda, University of Helsinki institutional repository.

This is an electronic reprint of the original article.

This reprint may differ from the original in pagination and typographic detail.

Please cite the original version. 


\title{
New Particle Formation from the Vapor Phase: From Barrier- Controlled Nucleation to the Collisional Limit
}

\author{
Kayane K. Dingilian, " Martina Lippe, Jakub Kubečka,, Jan Krohn, Chenxi Li, Roope Halonen, \\ Fatemeh Keshavarz, Bernhard Reischl, Theo Kurtén, Hanna Vehkamäki,* Ruth Signorell,* \\ and Barbara E. Wyslouzil*
}

Cite This: J. Phys. Chem. Lett. 2021, 12, 4593-4599

Read Online

ACCESS | Lلll Metrics \& More | 回 Article Recommendations | st Supporting Information

ABSTRACT: Studies of vapor phase nucleation have largely been restricted to one of two limiting cases - nucleation controlled by a substantial free energy barrier or the collisional limit where the barrier is negligible. For weakly bound systems, exploring the transition between these regimes has been an experimental challenge, and how nucleation evolves in this transition remains an open question. We overcome these limitations by combining complementary Laval expansion experiments, providing new particle formation data for carbon dioxide over a uniquely broad range of conditions. Our experimental data together with a kinetic model using rate constants from high-level quantum chemical calculations provide a comprehensive picture of new particle formation as nucleation transitions from a barrier-dominated process to the collisional limit.

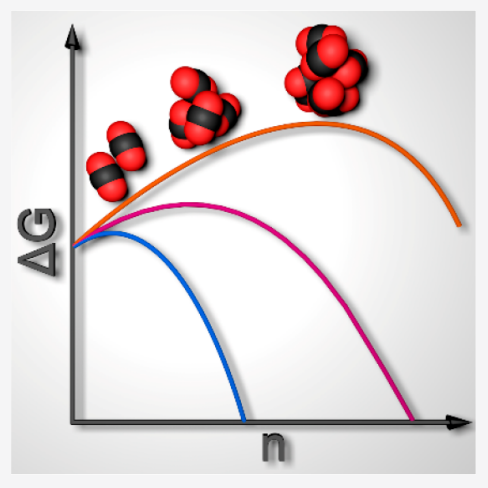

$\mathrm{N}$ ucleation from the vapor phase is the first step leading to new particle formation, giving it paramount importance in many atmospheric and technical processes. ${ }^{1-3}$ Understanding condensation of $\mathrm{CO}_{2}$ from the supersaturated vapor, for example, is important in processes ranging from cloud formation on Mars to $\mathrm{CO}_{2}$ recovery from flue gas or natural gas, via supersonic separation or cryogenic distillation. ${ }^{4}$ In the presence of pre-existing particles, condensation may be initiated by heterogeneous nucleation. In their absence, new particles are formed via homogeneous nucleation.

Under homogeneous conditions, new particle formation via nucleation is conceptually straightforward. Clusters are assumed to form and grow by monomer (single $\mathrm{CO}_{2}$ molecule) addition and decay by monomer loss. If the saturation ratio $S$ is large enough to ensure that cluster formation rates exceed cluster decay rates (i.e., $S>1$ and the vapor is supersaturated), new particles are produced. ${ }^{5}$ Here, $S$ $=p / p^{\text {eq }}$, where $p$ is the vapor pressure and $p^{\text {eq }}$ is the equilibrium vapor pressure above the appropriate condensed phase. Within the framework of nucleation theory, the cost of forming a surface between the existing metastable phase and the new stable phase introduces a free energy barrier that decreases in height with increasing supersaturation, ${ }^{6}$ and the barrier maximum occurs at the critical cluster size $n^{*}$. Once $S$ is high enough that the barrier vanishes, particle formation is largely governed by the kinetics of dimer formation. Such barrierless processes are not normally referred to as nucleation, but the term new particle formation is still appropriate.
Exploring the transition from nucleation controlled by a free energy barrier to nucleation in the barrier-free, collisional limit is, however, extremely challenging, because most nucleation experiments are optimized for a limited range of pressures and temperatures. Thus, experiments either probe conditions where the free energy barrier dominates or the collisional regime where reactive species, typically acids and bases, form extremely stable clusters. ${ }^{7-9}$ Recently, experiments have been developed to probe particle formation of weakly bound species, for example water, ${ }^{10-12} \mathrm{CO}_{2}{ }^{13}$ and various binary $\mathrm{CO}_{2}-$ containing mixtures, ${ }^{14}$ in the collisional limit under conditions of extreme supersaturation. To our knowledge, no experimental studies to date have explored the entire range from barrier-controlled nucleation to the collisional limit for a single system, and data in the transition regime are extremely limited. ${ }^{13}$ Theory and simulation ${ }^{15-19}$ have explored this regime more extensively, but questions remain on how well theory can capture the experimental trends in the transition region and in both limits.

The performance of theoretical approaches for predicting new particle formation rates depends mainly on the accuracy

Received: March 9, 2021

Accepted: April 19, 2021

Published: May 10, 2021 
with which the collision and evaporation rates can be calculated for clusters with sizes close to that of the critical cluster. When the process is fully kinetically controlled, i.e., evaporation of even the smallest clusters is negligible, the collision rate of two molecules determines the formation rate. The rates of collision and cluster growth can be calculated using the kinetic theory of gases, possibly corrected for longrange intermolecular forces. ${ }^{20}$ Cluster evaporation rates are considerably more difficult to calculate. One approach is to compute the cluster formation free energy using quantum chemistry (QC) and then calculate the evaporation rate using detailed balance (together with the collision rate). ${ }^{21}$ Unfortunately, the evaporation rates obtained with this approach depend exponentially on the free energies, and even small uncertainties in the latter can cause errors of several orders of magnitude in the former. Advances in computational power and recent improvements in computational chemistry have, however, helped overcome these obstacles. Key improvements include more accurate quantum mechanical methods, especially more affordable variants of the gold-standard coupled cluster method $\operatorname{CCSD}(\mathrm{T})$ and systematic approaches for configurational sampling of molecular clusters ${ }^{22}$ (SM, Section S3). Consequently, computational studies can now provide deep insight into the formation of complex molecular clusters, e.g., in the atmosphere. ${ }^{21}$ However, state-of-the-art computational methods have not previously focused on the exploration of the transition regime between barrierless particle formation and nucleation controlled by a free energy barrier.

We focus on particle formation of $\mathrm{CO}_{2}$ for two reasons. First, $\mathrm{CO}_{2}$ is an important and representative nonreactive species for particle formation via weakly bound clusters. Second, $\mathrm{CO}_{2}$ is a simple enough molecule for high-level quantum chemical calculations to estimate cluster free energies directly and predict nucleation rates from first-principles. Combining measurements within conventional supersonic Laval nozzles with those in the postnozzle flow lets us clearly observe a smooth transition from nucleation controlled by a barrier to the essentially barrier-free collisional limit. Both the more qualitative measures, i.e., the conditions at the onset of nucleation, and the quantitative measures, i.e., the nucleation rates, agree well in the region of overlap of the two experimental approaches. Furthermore, the good agreement between experiments and independent theoretical predictions based on QC calculations provides a molecular-level explanation of the observed trends and encouragement that nucleation predictions may one day be truly quantitative.

New particle formation in Laval nozzles. New particle formation was studied at The Ohio State University (OSU setup $)^{23-27}$ and ETH Zurich (ETH setup) ${ }^{12,13,28,29}$ (Figure 1, Section S1). Both experiments rely on supersonic Laval nozzle expansions to rapidly cool gas mixtures until temperatures are low enough to initiate homogeneous nucleation of $\mathrm{CO}_{2}$. In the continuously expanding OSU experiments, particles form in a short nucleation burst within the Laval nozzle. In the ETH experiments, new particles form throughout the uniform postnozzle flow. Combining results from the two experiments enables the study of new particle formation for the same molecule, as the process transitions from one controlled by a free energy barrier to one controlled by collision rates.

Static pressure $\left(p_{\mathrm{CO}_{2}}+p_{\mathrm{car}}\right), T$, and $S$ are rapidly changing functions of position within the OSU Laval nozzle. Once nucleation rates reach $\sim 10^{17} \mathrm{~cm}^{-3} \mathrm{~s}^{-1}$, particles form and grow

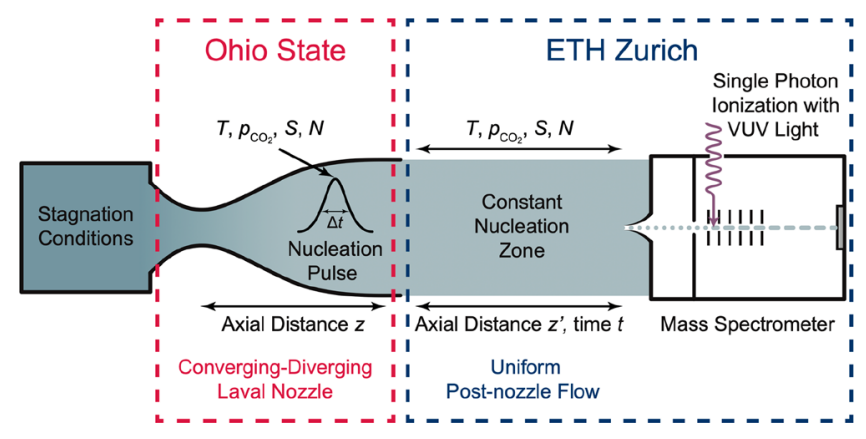

Figure 1. In the OSU (red) and the ETH setups (blue), the flow consists of $\mathrm{CO}_{2}\left(p_{\mathrm{CO}_{2}}\right)$ and carrier gas $\left(p_{\mathrm{car}}\right) . T, p_{\mathrm{CO}_{2}}, S$, and $N$ are the temperature, the partial pressure of $\mathrm{CO}_{2}$, the saturation ratio, and the particle number density, respectively, characterizing the experimental nucleation rate $J_{\exp }$. At OSU, static pressure measurements characterize the rapidly changing flow conditions $\left(T, p_{\mathrm{CO}_{2}}, S\right)$ and the nucleation pulse length, whereas small-angle X-ray scattering determines $N$. At ETH Zurich, impact pressure measurements characterize the stable postnozzle flow $\left(T, p_{\mathrm{CO}_{2}}, S\right.$ are constant), and nucleation is quantified by measuring cluster size distributions using soft single-photon ionization with vacuum ultraviolet (VUV) light coupled with mass spectrometry.

rapidly, releasing heat to the flow, increasing $T$ and static pressure, and in turn decreasing $S$ and quenching particle formation. Most particles are formed in a tight nucleation pulse that lasts $\sim 10 \mu \mathrm{s}$. The onset of nucleation is defined as the conditions at the maximum of the nucleation pulse (Section S1.A). These values, and the characteristic time $\Delta t$ associated with $J_{\text {exp }}$, are readily determined from the static pressure measurements. After the particle number density $N$ of the resultant aerosol is characterized via small-angle $\mathrm{X}$-ray scattering (SAXS), the nucleation rates are calculated using

$$
J_{\exp }=\frac{N}{\Delta t} \times \frac{\rho_{\mathrm{NZ}}}{\rho_{\mathrm{VV}}}
$$

where the density ratio $\rho_{\mathrm{NZ}} / \rho_{\mathrm{VV}}$ corrects for the continued expansion of the gas mixture between the nucleation zone (NZ) and the SAXS viewing volume (VV).

The ETH experiments measure nucleation in a uniform postnozzle flow where $T, p_{\mathrm{CO}_{2}}, p_{\text {car }}$, and $S$ are constant. Two types of experiments are conducted. The first characterizes the onset of nucleation by systematically changing the value of $T$ at a fixed $p_{\mathrm{CO}_{2}}$ and position $z^{\prime}$ until the transition from subcritical (no $\left(\mathrm{CO}_{2}\right)_{n}$ clusters are observed) to supercritical conditions $\left(\left(\mathrm{CO}_{2}\right)_{n}\right.$ clusters are observed $)$ is detected. A decrease in $T$ of $\sim 0.4 \mathrm{~K}$ is sufficient to observe this abrupt change. ${ }^{29}$ The second type of experiment records mass spectra at constant $S$ as a function of $z^{\prime}$ and, thus, of the nucleation time $t$. The total cluster concentration summed over all cluster sizes $n$ (number of molecules in the cluster $\left.\left(\mathrm{CO}_{2}\right)_{n}\right)$ greater than a critical size as a function of $t, N_{\text {cluster,tot }>n^{*}}$, directly yields the nucleation rate $J_{\exp }$ as $^{12}$

$$
J_{\text {exp }}=\frac{\partial N_{\text {cluster,tot }>n^{*}}(t)}{\partial t}
$$

The joint OSU/ETH onset data (this work and refs 13, 23, and 30) and existing literature values ${ }^{31,32}$ are summarized in Figure 2 in the form of a Volmer plot (Tables S1-S3 and Section S4). The good agreement between the OSU and ETH 


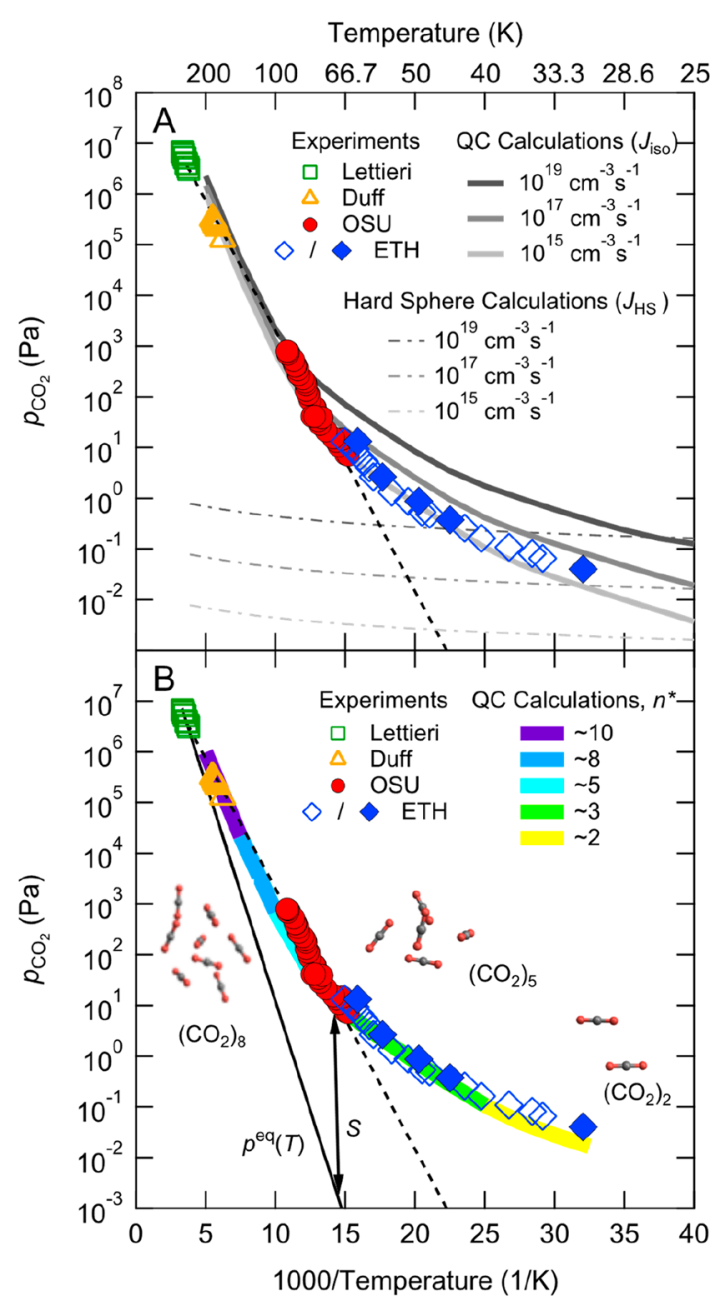

Figure 2. Experimental onset data from OSU (this work and ref 23), ETH ((open) this work and (filled) refs 13 and 30), Lettieri et al., ${ }^{32}$ and Duff. ${ }^{31}$ Filled symbols correspond to fully characterized nucleation rates (Figure 3). The agreement between the ETH and OSU values is very good in the overlap region. The $\log \left(p_{\mathrm{CO}_{2}}\right)$ required to initiate particle formation is a linear function of $1 / T$ from close to the critical point down to temperatures as low as $\sim 70 \mathrm{~K}$ (black dashed line). (A) Experimental onset data are consistent with nucleation rates of $10^{17 \pm 2} \mathrm{~cm}^{-3} \mathrm{~s}^{-1}$ obtained via the kinetic/quantum chemical model ( $J_{\text {iso, }}$ full lines). The latter merges smoothly with the hard sphere collision model neglecting evaporation $\left(J_{\mathrm{HS}}\right.$, dotted lines). (B) Critical cluster sizes $n^{*}$ corresponding to the experimental onset data conditions (Section S3.D) are estimated to lie between 2 and 8, and snapshots correspond to Gibbs-free-energy minimum structures. The equilibrium vapor pressure curve $p^{\text {eq }}(T)$ corresponds to liquid $\mathrm{CO}_{2}$. The deviation between the onset data and $p^{\mathrm{eq}}(T)$ illustrates the increase in supersaturation $S$ required to maintain a constant nucleation rate as $T$ decreases.

onset data in the overlap region (at $p_{\mathrm{CO}_{2}} \approx 13 \mathrm{~Pa}, T \approx 67-70$ $\mathrm{K})$ confirms the continuous trend from a linear behavior (dashed black line) of $\log \left(p_{\mathrm{CO}_{2}}\right)$ as a function of $1 / T$ at higher pressures and temperatures, to a flattening curve at lower pressures and temperatures. This reflects a transition from nucleation controlled by a roughly constant barrier to nucleation controlled by collision rates (vide infra). It is noteworthy that the data in Figure 2 cover 8 orders of magnitude in onset pressure and 1 order of magnitude in temperature with a large region that could only be covered by the joint OSU/ETH experiments. Nevertheless, the ideal gas assumption is still valid over the entire range of pressures and temperatures for which new particle formation rates were calculated.

The Volmer plot does not provide direct information about the experimental nucleation rates $J_{\text {exp }}$ an important quantitative measure for a comprehensive comparison (Figure 3).

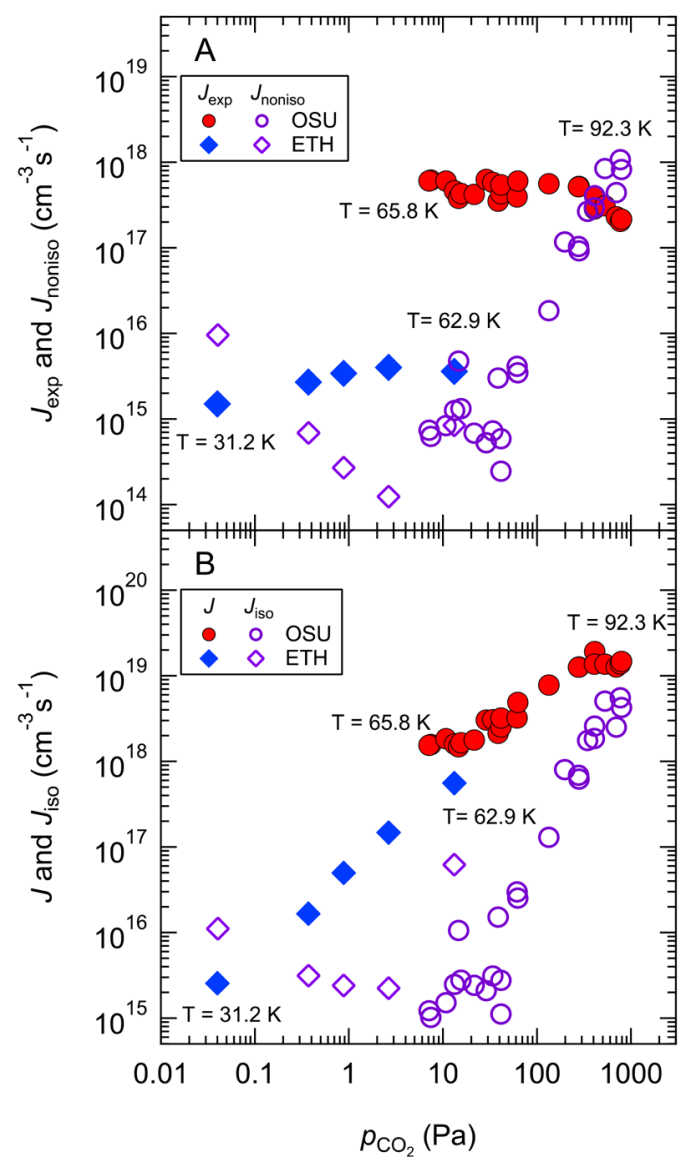

Figure 3. Experimental rates $\left(J_{\text {exp }}, J\right.$, Tables $S 1-S 3$ ) agree within $\sim 3$ orders of magnitude with rates calculated using QC methods $\left(J_{\text {noniso, }}\right.$ $J_{\text {iso }}$, Table S8). Effects of incomplete thermalization are accounted for (A) in $J_{\text {noniso }}$ by eq $S 15$ and for (B) in $J$ by eq 3 .

Comparing values of $J_{\exp }$ is, however, less straightforward than comparing onset data, because $J_{\exp }$ is more strongly influenced by the ratio of carrier gas to $\mathrm{CO}_{2}{ }^{33}$ In particular, the number of collisions between carrier gas molecules and nucleating $\mathrm{CO}_{2}$ clusters determines the degree of thermalization $\mathrm{CO}_{2}$ clusters achieve during nucleation. This, in turn, influences the nucleation rate, because poorly thermalized clusters are hotter and less stable than thermalized clusters. As the carrier gas to $\mathrm{CO}_{2}$ ratio decreases, lower nucleation rates are measured at the same system temperature and $p_{\mathrm{CO}_{2}}$. This explains the apparently poor agreement in the experimentally determined nucleation rates $J_{\exp }$ from OSU and ETH in the overlap region (Figure 3A; Section S2); rates recorded at roughly the same $\mathrm{CO}_{2}$ pressure $(\sim 10 \mathrm{~Pa})$ and similar temperatures $(T$ of 65.8 and $62.9 \mathrm{~K}$, respectively) differ by $\sim 2$ orders of magnitude. We note that in this region the OSU experiments use carrier gas to $\mathrm{CO}_{2}$ ratios of $\sim 200: 1$, whereas the ETH experiments operate at ratios of up to $1: 1$ (Table S3). At these $p_{\mathrm{CO}_{2}}$ values, 
nucleation rates are more strongly influenced by poor thermalization in the ETH experiments than in the OSU experiments. The OSU experiments are also affected by poor thermalization at higher $p_{\mathrm{CO}_{2}}$ where the carrier gas to $\mathrm{CO}_{2}$ ratio decreases to $\sim 2.5: 1$.

To account for the different degrees of thermalization-for a given experimental setup or between setups-we determined the nucleation rates $J$ expected under fully thermalized conditions by correcting $J_{\exp }$ with the factor $f$ derived by Feder et al. $^{33}$ (Section S1.C)

$$
J=J_{\exp } / f
$$

These values of $J$ at fixed $p_{\mathrm{CO}_{2}}$ and $T$ can now be compared directly, because they no longer depend on the carrier gas to $\mathrm{CO}_{2}$ ratio. Figure 3B shows that the agreement between the OSU and ETH values for $J$ at $p_{\mathrm{CO}_{2}} \approx 10 \mathrm{~Pa}$ is now almost perfect given the experimental uncertainties and a temperature difference of $\sim 3 \mathrm{~K}$. The correction closes the significant gap observed for $J_{\exp }$ (Figure 3A) almost completely and results in nucleation rates that increase continuously with increasing pressure, until they level off at the highest pressures (Figure 3B). The alignment of $J$ is equally good for results correlated by supersaturation (Figure S1B).

Modeling of new particle formation. Experimental observations are further supported by a kinetic model of particle formation that relies only on molecular properties. Cluster evaporation rates are determined using rate constants obtained from a combination of configurational sampling and high-level quantum chemical (QC) calculations (Section S3). Under the current conditions, only the addition or loss of single monomers need to be considered to calculate time-dependent cluster concentrations using the Becker-Döring model. ${ }^{34}$ Detailed balance is assumed for the association (monomer addition) and the evaporation (monomer loss) rate constants at $S=1$. Thus, evaporation rate constants are evaluated from cluster Gibbs free energies determined by accurate quantum chemical (QC) calculations (after configurational sampling of all relevant cluster structures) and association rate constants evaluated from gas kinetic theory (Section S3). With approximately constant monomer concentrations, the isothermal nucleation rate constant $J_{\text {iso }}$ is given by the steady-state solution of the rate equations (Section S3). Accounting for nonisothermal effects leads to $J_{\text {noniso }}$.

The methods used here allow us to access the largely unexplored transition between these two limiting regimes, independently via experiments and calculations (vide infra). With their exponential dependence on cluster formation free energies, nucleation rates offer one of the most direct and sensitive comparisons between experiment and QC-based modeling. Figure 3 reveals a very good agreement between the experimental results and the theoretical predictions (Table S8) over the entire range of experimentally determined nucleation rates of $J \approx 10^{15}-10^{19} \mathrm{~cm}^{-3} \mathrm{~s}^{-1}$, with differences of at most $\sim 3$ orders of magnitude, for both the nonisothermal (Figure $3 \mathrm{~A}$ ) and the isothermal (Figure 3B) rates. The $\mathrm{QC}$ data has a general accuracy of a few $k_{\mathrm{B}} T$ leading to a rough uncertainty of 1 order of magnitude in $J_{\text {iso }}$. Additional errors may arise from insufficiently accounting for thermal contributions from all existing cluster configurations and the assumption that all vibrations are (quasi-)harmonic and uncoupled, which might not be completely valid, as the small $\mathrm{CO}_{2}$ clusters may behave in a liquid-like manner at the studied temperatures. ${ }^{35}$ Given the complexity of nucleation processes and the very high sensitivity of nucleation rates to system parameters, such good agreement is extremely encouraging. This is especially true in light of the 1 order of magnitude uncertainties in the absolute values of the experimental rates and the fact that the theoretical predictions are essentially free of adjustable parameters and made independently of the experimental results.

From nucleation to the collision limit. While nucleation rates are highly sensitive to local conditions, their value alone does not provide information on the nucleation regime, i.e., high barrier limit, collisional limit, or transition regime. Comparing experimental onset data to model predictions (Figure 2A) offers a way forward. The change in the trend of the Volmer plot, from a linear decrease of $\log \left(p_{\mathrm{CO}_{2}}\right)$ (dashed black line) with $1 / T$ at higher pressures and temperatures to a curve flattening at lower pressures and temperatures clearly suggests a change in nucleation regime. The experimental trends in Figure $2 \mathrm{~A}$ are captured by model predictions for rates that cover the same range $\left(10^{17 \pm 2} \mathrm{~cm}^{-3} \mathrm{~s}^{-1}\right)$, using the kinetic/QC

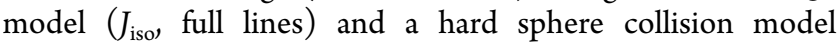
neglecting evaporation ( $J_{\mathrm{HS}}$, dotted lines, see Section S3.A). The agreement between the experimental data, $J_{\text {iso }}$ and $J_{\mathrm{HS}}$ for the coldest vapor $(T \approx 30 \mathrm{~K})$ indicates that the collision limit is approached at the highest supersaturations. This is qualitatively reflected in the very low nucleation barrier calculated from QC (Figure 4) and a very small critical size $n^{*}$ (Figure $2 \mathrm{~B}$ and Table S8). At $T=31.2 \mathrm{~K}$, the nucleation barrier $\Delta G^{*}$ is $\sim 4 k_{\mathrm{B}} T$ or $\sim 1 \mathrm{~kJ} / \mathrm{mol}$, roughly the accuracy of the $\mathrm{QC}$ calculations, which are thus consistent with essentially collision-limited nucleation, i.e., $\Delta G^{*}$ and $n^{*}$ on the order of $k_{\mathrm{B}} T$ and 1 , respectively.

The large and rapid deviation of the experimental data from $J_{\mathrm{HS}}$ as the temperature rises above $\sim 70 \mathrm{~K}$ (Figure $2 \mathrm{~A}$ ) indicates that nucleation is now dominated by the presence of a substantial free energy barrier. At $T \approx 92.3 \mathrm{~K}$, for example, $\Delta G^{*}$ is $\sim 15 k_{\mathrm{B}} T$ or $\sim 13 \mathrm{~kJ} / \mathrm{mol}$ - a value much larger than the accuracy of the QC calculations-and $n^{*}$ is $\sim 5$, clearly indicating barrier-limited nucleation. Furthermore, the linear behavior of the data observed in the temperature range between $\sim 70-90 \mathrm{~K}$ coincides with the appearance of an approximately constant $\Delta G^{*} / k_{\mathrm{B}} T$ (Section S4, eq S22: at a large enough $n^{*}$, the functional form of the temperature dependence largely reflects that of $\left.p^{\mathrm{eq}}(T)\right)$. The QC calculations show that the nucleation barrier becomes broader (Figure $4 \mathrm{~A}$ ) but not higher in this range (see Figure 4B region with $\Delta G^{*} / k_{\mathrm{B}} T \approx$ constant above $p_{\mathrm{CO}_{2}} \approx 100 \mathrm{~Pa}$ ). The linear behavior extends to even lower saturations (higher temperatures, green squares and yellow triangles in Figure 2A), with a generally very minor dependence on the value of the nucleation rate (almost overlapping $J_{\text {iso }}$ curves), again explicable by classical nucleation theory (Section S4).

The theoretical results at high and low saturation confirm that the measurements for temperatures between $\sim 40$ and 70 $\mathrm{K}$ indeed probe the transition regime. The $\mathrm{QC}$ calculations predict that $\Delta G^{*} / k_{\mathrm{B}} T$ increases steeply with decreasing supersaturation (Figure $4 \mathrm{~B}$ ) as does $n^{*}$ starting from close to unity (Figure 2B), i.e., with decreasing saturation, we see a transition from collisional-controlled to barrier-controlled nucleation. The agreement between experiment and the independent theoretical prediction for both the onset (Figure 2A) and rate (Figure 3) data is encouraging. For $\mathrm{CO}_{2}$, the trend in the onset data can also be captured by classical 

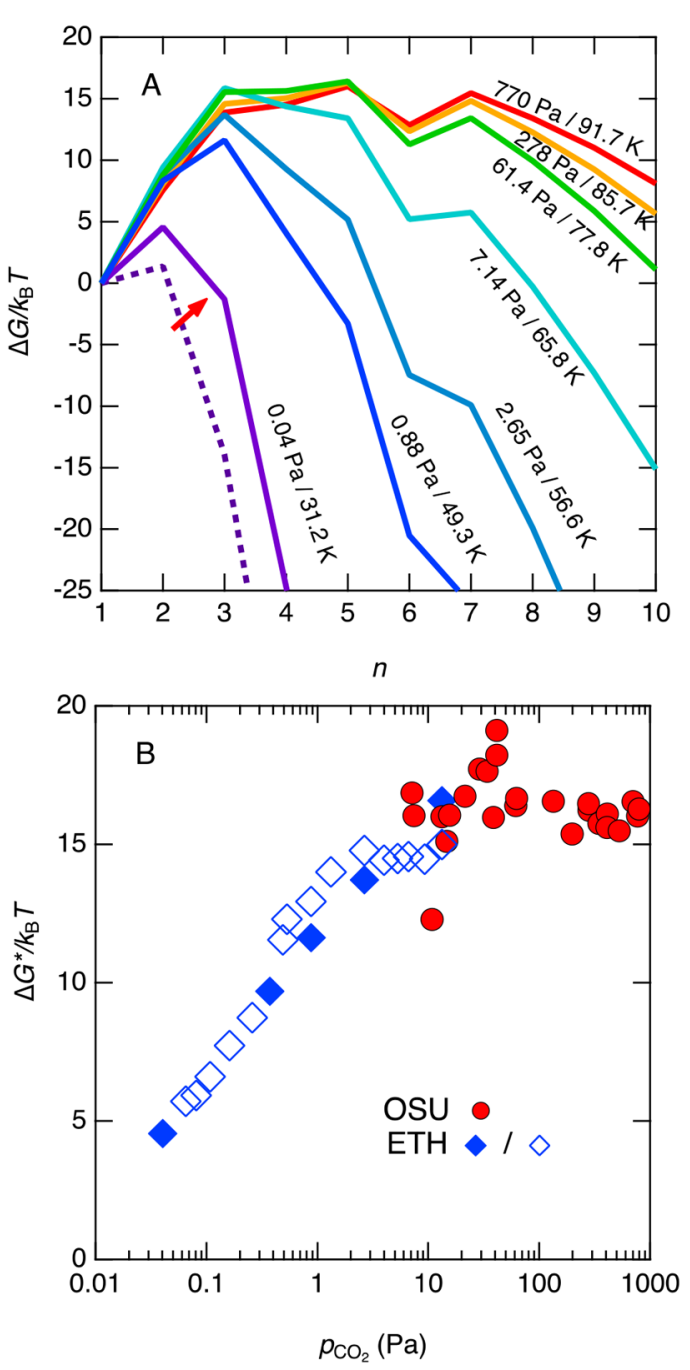

Figure 4. (A) Free energy barriers $\Delta G(n) / k_{\mathrm{B}} T$ for the noted experiment conditions (Tables S1-S3). The dashed line corresponds to $\Delta G(n) / k_{\mathrm{B}} T$ calculated at the lower level of theory for $p_{\mathrm{CO}_{2}}=0.04$ $\mathrm{Pa}$ (Section S3.C and Table S8). The barrier broadens as its height increases. (B) Barrier heights $\Delta G^{*} / k_{\mathrm{B}} T$ based on $\mathrm{QC}$ calculations for all experimental conditions increase rapidly with $p_{\mathrm{CO}_{2}}$, leveling off for $p_{\mathrm{CO}_{2}}>\sim 10 \mathrm{~Pa}$. Filled symbols indicate conditions for which nucleation rates are measured.

nucleation theory with suitably chosen parameters (Figure S3). But, as detailed extensively in Dingilian et al., ${ }^{23}$ the agreement with the experiments depends sensitively not only on the substance but also on (1) the choice of theory, (2) the choice of physical property values, and (3) an assumption regarding the state of the critical cluster ("liquid-like" or "crystalline"). ${ }^{23}$ For the range of physical properties and classical nucleation rate expressions investigated there, the ratio of measured to predicted nucleation rates varied from $10^{-6}$ to $10^{18}$. While reliable a priori predictions of experimental nucleation rates can hardly be expected from any variant of classical nucleation theory, the QC-based kinetics approach of the present work was able to predict the conditions under which nucleation is plausible and quantify the nucleation rates to within a few orders of magnitude.

Outlook. The present study shows that new particle formation can be understood over the entire range of possible nucleation mechanisms by combining experimental data and QC-based kinetic modeling. The Volmer plot shows characteristic trends, from a linear behavior where the barrier is substantial to an increasingly flattened curve that extends from the transition regime to the collisional limit. Starting from the collisional limit, the barrier height, critical cluster size, and nucleation rate increase with decreasing supersaturation and plateau in the region of barrier-dominated nucleation.

For many applications where nucleation plays a key role, reliable theoretical predictions of the conditions and rates at which nucleation occurs would be tremendously useful but are largely missing. The $\mathrm{CO}_{2}$ example presented here demonstrates the predictive power of the combined QC kinetics approach-not only for a special regime but over the entire range of nucleation behaviors. Future studies will have to explore how far the predictive power of the present model can be extended to other systems. Be it from the experimental or from the theoretical side, the question whether general trends over different regions of nucleation behavior will also occur in multicomponent systems will be an exciting route to followparticularly for systems that show nucleation enhancement or suppression. ${ }^{7,14,20}$

\section{ASSOCIATED CONTENT}

\section{Supporting Information}

The Supporting Information is available free of charge at https://pubs.acs.org/doi/10.1021/acs.jpclett.1c00762.

Additional information about the experiments and the calculations are provided in Sections S1-S4, Tables S1S8, and Figures S1-S3 (PDF)

\section{AUTHOR INFORMATION}

\section{Corresponding Authors}

Hanna Vehkamäki - Institute for Atmospheric and Earth System Research/Physics, University of Helsinki, FI-00014 Helsinki, Finland; $\odot$ orcid.org/0000-0002-5018-1255; Email: hanna.vehkamaki@helsinki.fi

Ruth Signorell - Department of Chemistry and Applied Biosciences, ETH Zürich, 8093 Zürich, Switzerland; ○ orcid.org/0000-0003-1111-9261; Email: rsignorell@ ethz.ch

Barbara E. Wyslouzil - William G. Lowrie Department of Chemical and Biomolecular Engineering and Department of Chemistry and Biochemistry, The Ohio State University, Columbus, Ohio 43210, United States; (1) orcid.org/00000001-9763-5990; Email: wyslouzil.1@osu.edu

\section{Authors}

Kayane K. Dingilian - William G. Lowrie Department of Chemical and Biomolecular Engineering, The Ohio State University, Columbus, Ohio 43210, United States

Martina Lippe - Department of Chemistry and Applied Biosciences, ETH Zürich, 8093 Zürich, Switzerland

Jakub Kubečka - Institute for Atmospheric and Earth System Research/Physics, University of Helsinki, FI-00014 Helsinki, Finland; O orcid.org/0000-0002-8002-0911

Jan Krohn - Department of Chemistry and Applied Biosciences, ETH Zürich, 8093 Zürich, Switzerland

Chenxi Li - Department of Chemistry and Applied Biosciences, ETH Zürich, 8093 Zürich, Switzerland 
Roope Halonen - Institute for Atmospheric and Earth System Research/Physics, University of Helsinki, FI-00014 Helsinki, Finland

Fatemeh Keshavarz - Institute for Atmospheric and Earth System Research/Physics, University of Helsinki, FI-00014 Helsinki, Finland; (1) orcid.org/0000-0003-2189-7809

Bernhard Reischl - Institute for Atmospheric and Earth System Research/Physics, University of Helsinki, FI-00014 Helsinki, Finland; (i) orcid.org/0000-0001-7333-4923

Theo Kurtén - Department of Chemistry, Faculty of Science, University of Helsinki, FI-00014 Helsinki, Finland; (1) orcid.org/0000-0002-6416-4931

Complete contact information is available at: https://pubs.acs.org/10.1021/acs.jpclett.1c00762

\section{Author Contributions}

R.S. and B.E.W. conceptualized the work, led the experiments, and wrote and edited the paper together with contributions from K.K.D., M.L., J.Ku., J.Kr., R.H., and H.V. H.V. coordinated and supervised the computational work. T.K. ensured the consistency of the quantum chemical methods used. K.K.D. conducted the OSU experiments and analyzed data with experimental contributions from M.L. M.L., J.Kr., and C.L. conducted the ETH experiments analyzed data. J.Ku. performed the configurational sampling, some of the lowerlevel QC calculations, and participated in the kinetic modeling. R.H. worked on the kinetic model and classical theory and derived some analytical expressions for the QC nonisothermal correction. B.R. tested and selected the force field parameters. F.K. performed the highest accuracy QC calculations. All coauthors have read the paper.

\section{Author Contributions}

${ }^{\#}$ K.K.D., M.L., and J.Ku. contributed equally.

\section{Notes}

The authors declare no competing financial interest.

Data Repository: All data needed to evaluate the conclusions of the paper are presented in the paper, the Supporting Information, or are deposited on the ETH Research Collection under DOI: https://doi.org/10.3929/ethz-b-000462780. Additional data related to this paper may be requested from the authors.

\section{ACKNOWLEDGMENTS}

Financial support was provided by the Swiss National Science Foundation (SNSF project numbers 200020-172472 and 200020_200306 and the NCCR MUST), ETH Zürich, National Science Foundation (NSF Grants CHE-1464924, CHE-1900064), European Research Council project 692891DAMOCLES, Academy of Finland and the University of Helsinki (Faculty of Science ATMATH project). The CSC Finnish IT Centre provided access to computer clusters, with computer capacity from the Finnish Grid and Cloud Infrastructure (persistent identifier urn:nbn:fi:research-infras2016072533). R.S. gratefully acknowledges a Humboldt Research Prize (Alexander von Humboldt Foundation) and a Mildred Dresselhaus Award (Centre for Ultrafast Imaging in Hamburg). B.E.W. acknowledges a Guest Professorship from ETH Zürich.

\section{REFERENCES}

(1) Young, J. B.; Yau, K. K.; Walters, P. T. Fog Droplet Deposition and Coarse Water Formation in Low-Pressure Steam Turbines: A
Combined Experimental and Theoretical Analysis. J. Turbomach. $1988,110,163-172$.

(2) Lee, S.-H.; Gordon, H.; Yu, H.; Lehtipalo, K.; Haley, R.; Li, Y.; Zhang, R. New Particle Formation in the Atmosphere: From Molecular Clusters to Global Climate. J. Geophys. Res.: Atmos. 2019, 124, 7098-7146.

(3) Nachbar, M.; Duft, D.; Mangan, T. P.; Martin, J. C. G.; Plane, J. M. C.; Leisner, T. Laboratory Measurements of Heterogeneous $\mathrm{CO}_{2}$ Ice Nucleation on Nanoparticles Under Conditions Relevant to the Martian Mesosphere. J. Geophys. Res. Atmos. 2016, 121, 753-769.

(4) Hart, A.; Gnanendran, N. Cryogenic $\mathrm{CO}_{2}$ Capture in Natural Gas. Energy Procedia 2009, 1, 697-706.

(5) Li, C.; Signorell, R. Understanding Vapor Nucleation on the Molecular Level: A Review. J. Aerosol Sci. 2021, 153, 105676.

(6) Vehkamäki, H. Classical Nucleation Theory in Multicomponent Systems; Springer Science \& Business Media: Berlin, 2006.

(7) Dunne, E. M.; Gordon, H.; Kürten, A.; Almeida, J.; Duplissy, J.; Williamson, C.; Ortega, I. K.; Pringle, K. J.; Adamov, A.; Baltensperger, U.; Barmet, P.; Benduhn, F.; Bianchi, F.; Breitenlechner, M.; Clarke, A.; Curtius, J.; Dommen, J.; Donahue, N. M.; Ehrhart, S.; Flagan, R. C.; Franchin, A.; Guida, R.; Hakala, J.; Hansel, A.; Heinritzi, M.; Jokinen, T.; Kangasluoma, J.; Kirkby, J.; Kulmala, M.; Kupc, A.; Lawler, M. J.; Lehtipalo, K.; Makhmutov, V.; Mann, G.; Mathot, S.; Merikanto, J.; Miettinen, P.; Nenes, A.; Onnela, A.; Rap, A.; Reddington, C. L. S.; Riccobono, F.; Richards, N. A. D.; Rissanen, M. P.; Rondo, L.; Sarnela, N.; Schobesberger, S.; Sengupta, K.; Simon, M.; Sipilä, M.; Smith, J. N.; Stozkhov, Y.; Tomé, A.; Tröstl, J.; Wagner, P. E.; Wimmer, D.; Winkler, P. M.; Worsnop, D. R.; Carslaw, K. S. Global Atmospheric Particle Formation From CERN CLOUD Measurements. Science 2016, 354, 1119-1124.

(8) Jen, C. N.; McMurry, P. H.; Hanson, D. R. Stabilization of Sulfuric Acid Dimers by Ammonia, Methylamine, Dimethylamine, and Trimethylamine. J. Geophys. Res. Atmos. 2014, 119, 7502-7514.

(9) Chen, H.; Ezell, M. J.; Arquero, K. D.; Varner, M. E.; Dawson, M. L.; Gerber, R. B.; Finlayson-Pitts, B. J. New Particle Formation and Growth From Methanesulfonic Acid, Trimethylamine and Water. Phys. Chem. Chem. Phys. 2015, 17, 13699-13709.

(10) Bourgalais, J.; Roussel, V.; Capron, M.; Benidar, A.; Jasper, A. W.; Klippenstein, S. J.; Biennier, L.; Le Picard, S. D. Low Temperature Kinetics of the First Steps of Water Cluster Formation. Phys. Rev. Lett. 2016, 116, 113401.

(11) Lippe, M.; Chakrabarty, S.; Ferreiro, J. J.; Tanaka, K. K.; Signorell, R. Water Nucleation at Extreme Supersaturation. J. Chem. Phys. 2018, 149, 244303.

(12) Li, C.; Lippe, M.; Krohn, J.; Signorell, R. Extraction of Monomer-Cluster Association Rate Constants From Water Nucleation Data Measured at Extreme Supersaturations. J. Chem. Phys. 2019, $151,094305$.

(13) Krohn, J.; Lippe, M.; Li, C.; Signorell, R. Carbon Dioxide and Propane Nucleation: The Emergence of a Nucleation Barrier. Phys. Chem. Chem. Phys. 2020, 22, 15986-15998.

(14) Li, C.; Krohn, J.; Lippe, M.; Signorell, R. How Volatile Components Catalyze Vapor Nucleation. Sci. Adv. 2021, 7, No. eabd9954.

(15) Bhimalapuram, P.; Chakrabarty, S.; Bagchi, B. Elucidating the Mechanism of Nucleation Near the Gas-Liquid Spinodal. Phys. Rev. Lett. 2007, 98, 206104.

(16) Mendez-Villuendas, E.; Saika-Voivod, I.; Bowles, R. K. A Limit of Stability in Supercooled Liquid Clusters. J. Chem. Phys. 2007, 127, 154703.

(17) Wedekind, J.; Chkonia, G.; Wölk, J.; Strey, R.; Reguera, D. Crossover From Nucleation to Spinodal Decomposition in a Condensing Vapor. J. Chem. Phys. 2009, 131, 114506.

(18) Santra, M.; Bagchi, B. Crossover Dynamics at Large Metastability in Gas-Liquid Nucleation. Phys. Rev. E 2011, 83, 031602 .

(19) Singh, R. S.; Santra, M.; Bagchi, B. Sensitivity of Nucleation Phenomena on Range of Interaction Potential. J. Chem. Phys. 2012, $136,084701$. 
(20) Halonen, R.; Zapadinsky, E.; Kurtén, T.; Vehkamäki, H.; Reischl, B. Rate Enhancement in Collisions of Sulfuric Acid Molecules Due to Long-Range Intermolecular Forces. Atmos. Chem. Phys. 2019, 19, 13355-13366.

(21) Elm, J.; Kubečka, J.; Besel, V.; Jääskeläinen, M. J.; Halonen, R.; Kurtén, T.; Vehkamäki, H. Modeling the Formation and Growth of Atmospheric Molecular Clusters: A Review. J. Aerosol Sci. 2020, 149, 105621 .

(22) Kubečka, J.; Besel, V.; Kurtén, T.; Myllys, N.; Vehkamäki, H. Configurational Sampling of Noncovalent (Atmospheric) Molecular Clusters: Sulfuric Acid and Guanidine. J. Phys. Chem. A 2019, 123, 6022-6033.

(23) Dingilian, K. K.; Halonen, R.; Tikkanen, V.; Reischl, B.; Vehkamäki, H.; Wyslouzil, B. E. Homogeneous Nucleation of Carbon Dioxide in Supersonic Nozzles I: Experiments and Classical Theories. Phys. Chem. Chem. Phys. 2020, 22, 19282-19298.

(24) Tanimura, S.; Park, Y.; Amaya, A.; Modak, V.; Wyslouzil, B. E. Following Heterogeneous Nucleation of $\mathrm{CO}_{2}$ on $\mathrm{H}_{2} \mathrm{O}$ Ice Nanoparticles With Microsecond Resolution. RSC Adv. 2015, 5, 105537105550.

(25) Park, Y.; Wyslouzil, B. E. $\mathrm{CO}_{2}$ Condensation Onto Alkanes: Unconventional Cases of Heterogeneous Nucleation. Phys. Chem. Chem. Phys. 2019, 21, 8295-8313.

(26) Ogunronbi, K. E.; Sepehri, A.; Chen, B.; Wyslouzil, B. E. Vapor Phase Nucleation of the Short-Chain $n$-Alkanes ( $n$-Pentane, $n$-Hexane and $n$-Heptane): Experiments and Monte Carlo Simulations. J. Chem. Phys. 2018, 148, 144312.

(27) Ogunronbi, K. E.; Wyslouzil, B. E. Vapor-Phase Nucleation of $n$-Pentane, $n$-Hexane, and $n$-Heptane: Critical Cluster Properties. J. Chem. Phys. 2019, 151, 154307.

(28) Schläppi, B.; Litman, J. H.; Ferreiro, J. J.; Stapfer, D.; Signorell, R. A Pulsed Uniform Laval Expansion Coupled With Single Photon Ionization and Mass Spectrometric Detection for the Study of Large Molecular Aggregates. Phys. Chem. Chem. Phys. 2015, 17, 2576125771 .

(29) Chakrabarty, S.; Ferreiro, J. J.; Lippe, M.; Signorell, R. Toluene Cluster Formation in Laval Expansions: Nucleation and Growth. J. Phys. Chem. A 2017, 121, 3991-4001.

(30) Lippe, M.; Szczepaniak, U.; Hou, G.-L.; Chakrabarty, S.; Ferreiro, J. J.; Chasovskikh, E.; Signorell, R. Infrared Spectroscopy and Mass Spectrometry of $\mathrm{CO}_{2}$ Clusters During Nucleation and Growth. J. Phys. Chem. A 2019, 123, 2426-2437.

(31) Duff, K. M. Non-Equilibrium Condensation of Carbon Dioxide in Supersonic Nozzles. Thesis, Massachusetts Institute of Technology, 1966.

(32) Lettieri, C.; Paxson, D.; Spakovszky, Z.; Bryanston-Cross, P. Characterization of Nonequilibrium Condensation of Supercritical Carbon Dioxide in a De Laval Nozzle. J. Eng. Gas Turbines Power 2018, 140, 041701.

(33) Feder, J.; Russell, K. C.; Lothe, J.; Pound, G. M. Homogeneous Nucleation and Growth of Droplets in Vapours. Adv. Phys. 1966, 15, 111-178.

(34) Becker, R.; Döring, W. Kinetische Behandlung der Keimbildung in übersättigten Dämpfen. Ann. Phys. (Berlin, Ger.) 1935, 416, 719752.

(35) Halonen, R.; Tikkanen, V.; Reischl, B.; Dingilian, K. K.; Wyslouzil, B. E.; Vehkamäki, H. Homogeneous Nucleation of Carbon Dioxide in Supersonic Nozzles II: Molecular Dynamics Simulations and Properties of Nucleating Clusters. Phys. Chem. Chem. Phys. 2021, 23, 4517-4529. 